\title{
Noble Metal Nanostructures Influence of Structure and Environment on Their Optical Properties
}

\author{
Ondřej Kvítek, ${ }^{1}$ Jakub Siegel, ${ }^{1}$ Vladimír Hnatowicz, ${ }^{2}$ and Václav Švorčík ${ }^{1}$ \\ ${ }^{1}$ Department of Solid State Engineering, Institute of Chemical Technology, 16628 Prague, Czech Republic \\ ${ }^{2}$ Nuclear Physics Institute, Academy of Sciences of the Czech Republic, 25068 Rez, Czech Republic \\ Correspondence should be addressed to Ondřej Kvítek; kviteko@vscht.cz
}

Received 21 March 2013; Accepted 9 August 2013

Academic Editor: Christian Brosseau

Copyright (c) 2013 Ondřej Kvítek et al. This is an open access article distributed under the Creative Commons Attribution License, which permits unrestricted use, distribution, and reproduction in any medium, provided the original work is properly cited.

\begin{abstract}
Optical properties of nanostructured materials, isolated nanoparticles, and structures composed of both metals and semiconductors are broadly discussed. Fundamentals of the origin of surface plasmons as well as the surface plasmon resonance sensing are described and documented on a number of examples. Localized plasmon sensing and surface-enhanced Raman spectroscopy are subjected to special interest since those techniques are inherently associated with the direct application of plasmonic structures. The possibility of tailoring the optical properties of ultra-thin metal layers via controlling their shape and morphology by postdeposition annealing is documented. Special attention is paid to the contribution of bimetallic particles and layers as well as metal structures encapsulated in semiconductors and dielectrics to the optical response. The opportunity to tune the properties of materials over a large scale of values opens up entirely new application possibilities of optical active structures. The nature of surface plasmons predetermines noble metal nanostructures to be promising great materials for development of modern label-free sensing methods based on plasmon resonance-SPR and LSPR sensing.
\end{abstract}

\section{Introduction}

The crucial influence of the fine structure of materials on various mechanical, magnetic, and electronic properties was recognized at the end of 19th century. This line of thought seems to start with the pioneering work on the mechanical properties of iron alloys. These studies led to the conclusion that fine-scale microstructure retained after allotropic transformation of iron alloys gives martensite its hardness. The discovery of precipitation hardening in 1906 was the first observation suggesting that the correlation between microstructure and properties (originally proposed for ferrous alloys only) applies to nonferrous materials as well. Numerous observations in the subsequent years substantiated and generalized this view and led to the classification of the properties of solids with different types of chemical bonding into microstructure-sensitive and nonmicrostructure-sensitive ones. The physical understanding of the mechanisms by which ultrafine microstructures affect the properties of solids received a remarkable boost after the advent of the theory of lattice defects and the availability of new high-resolution characterization techniques such as electron and field ion microscopy. Both developments helped to elucidate the physical basis for understanding the correlation between the structure-sensitive properties and the microstructure of solids. Second period of developments in the area of nanomaterials started, when it was recognized that modification of the structure leads to generation of new atomic and electronic structures in the solids [1].

Bulk noble metals are known for their high thermal and electrical conductivity, specific mechanical properties, and high reflectivity of incident radiation. These properties are caused by their crystalline structure and presence of delocalized electrons-existence of electron gas. With decreasing thicknesses of metal films, new properties of the material can be observed. Optical properties are no exception, and a great shift can be seen in the appearance of a material of the same composition but different nanostructure. For example bulk gold is known as a shiny, yellow noble metal that does not tarnish. Smooth, thin films of the same metal appear opaque, and nanostructured surface covered with gold islands appears 


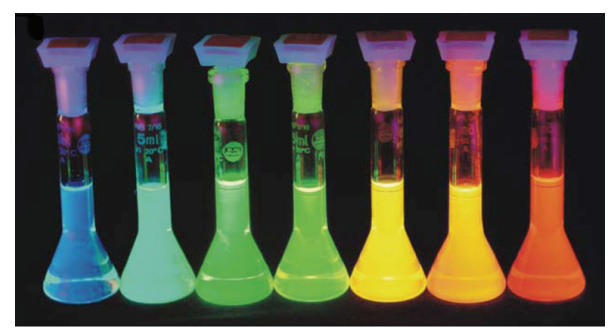

(a)

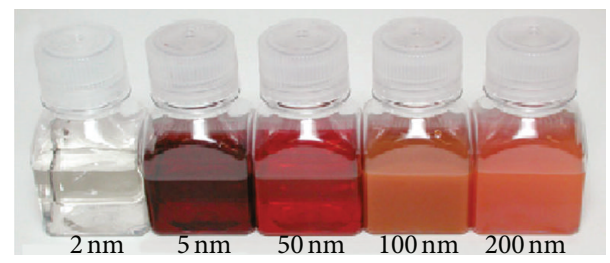

(b)

Figure 1: (a) Fluorescence of CdSe-CdS core-shell nanoparticles with a diameter of $1.7 \mathrm{~nm}$ (blue) up to $6 \mathrm{~nm}$ (red). (b) Size dependence of gold colloid optical absorption properties [2,3].

transparent with color changing from blue to red or even green depending on the structure size. Thus we can say that simply by changing the particle size or morphology of the material retaining its chemical composition, we can obtain structures with dramatically different properties. This property tuning by controlling the nanostructure of materials has proven to be very useful in specific applications.

\section{Principles}

A good example of the tuning of the electronic structure of a material by modifying structural properties is CdS nanoparticles. By controlling the size of these semiconducting nanoparticles in the range of $2-5 \mathrm{~nm}$, band gap of the material can be adjusted between 4.5 and $2.5 \mathrm{eV}$ [4]. The decreasing radius of the nanoparticles results in an increase of the band gap width with a decrease of the valence band maximum and an increase of the conductive band minimum, both contributing equally. This is very well displayed in the fluorescence color of the colloid solution (Figure 1(a)). It is normally considered that clusters of about 200 atoms have already the same unit cell and bond lengths as bulk material, but in the case of II-VI semiconductors, such as CdS, at least $10^{4}$ atoms are needed for the bulk behaviour to fully develop [5]. This is caused by a high ratio of the surface atoms in particles of this size. Increased influence of the irregular surface with high density of kinks, edges, and corners leads to presence of surface states which can trap electrons and holes and change optical and electronic properties of the material considerably. Shifts of band positions with varying particle size in optical absorption spectra of metallic nanoparticles resemble those of semiconductor clusters (compare Figures 1(a) and 1(b)). The origin of the resonant absorption in this case may, however, be quite different. Absorption due to electron interband transitions often roleplay (with decreasing size of a metal particle, band gap can be formed leading to transition of the properties of the material from metal to semiconductor or insulator), but the present delocalized conduction electrons which can be excited collectively into surface plasmons have also great influence on the mechanism of evolution and origin of certain absorption bands in the spectra [6].

2.1. Surface Plasmons and Surface Plasmon Resonance Sensing $(S P R)$. We have already stated that decreasing the size of the basic structural elements leads, among others, to changes of optical response of a material. A great deal of these changes is caused by increasing influence of certain electromagnetic surface modes-coherent fluctuations of electron charges on metal boundary called surface plasma oscillations or plasmons. Excitation of surface plasmons takes place, when the surface of the metal is exposed to incoming electrons or photons. Plasmons are strongly bound to the incident surface with their maximum intensity at the surface and disappear quickly with increasing distance from the surface. Therefore they are very sensitive to the surface properties. For photons to excite plasmons in the smooth metal surface, application of grating or attenuated total reflection (ATR) coupling is necessary to accomplish the need for appropriate wave vectors of the excited surface plasmon. On rough surfaces as well as gratings of appropriate parameters, surface plasmons can be reversely transformed into light. This light emission can play an important role in characterizing the roughness of the metal surface [7].

The propagation length of the surface plasmons in the metal surface as well as in surrounding medium is determined by their dissipation due to the internal and radiation damping. Internal damping is caused by excitation of electron-hole pairs at the Fermi level of the metal with following deexcitation producing phonons and thus heating the material (this process can be observed by photoacoustic measurements) and emission of the photoelectrons. Radiation damping consists in transformation of the evanescent wave of the surface plasmon into a plane wave. This process is characteristic of two-interface system such as the ATR device. The arrangement using ATR coupling to excite surface plasmons is nowadays well known and used in detection devices that evaluate the dependence of the incidence angle at which the surface plasmon resonance reaches its maximum (when frequency of coupled photons matches the frequency of the collective electron oscillations) on the medium surrounding the thin metal film (Figure 2). SPR detection methods prove to be very useful to study biological interactions in their natural state (or as close as possible), as there is, compared to standard methods of detection, no need for labeling. Labels can affect interactions between the biomolecules, so label-free techniques are preferred. However, there are also problems with SPR sensors in comparison to other types of detection devices (mainly immunoassays), because of nothigh-enough sensitivity of the SPR sensors and the necessity to integrate them into a complicated optic system to excite the surface plasmons, which hinders their application in common laboratory arrangements. There is a number of 


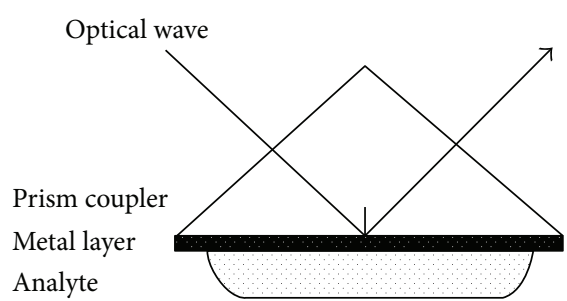

(a)

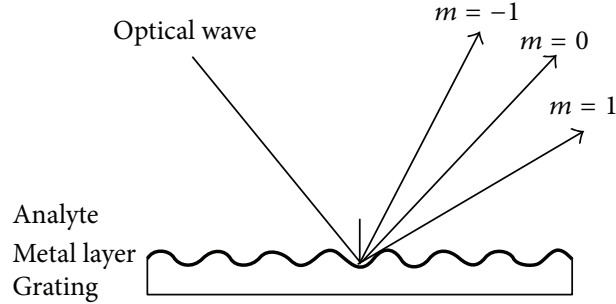

(b)

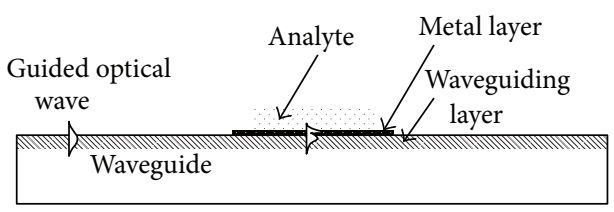

(c)

FIGURE 2: Most widely used configurations of SPR sensors: (a) prism coupler-based SPR system (ATR method); (b) grating coupler-based SPR system; (c) optical waveguide-based SPR system [8].

recent reviews particularly devoted to the field of SPR sensing and biosensing $[8,16,17]$.

\subsection{Localized Plasmons and Surface-Enhanced Raman Spec-} troscopy (SERS). To eliminate the need for complicated coupling optics, nanostructured layers of isolated islands can be employed. In these structures, it is possible to excite surface plasmons by simple usage of incoming radiation with no need for ATR coupling or gratings, thanks to the naturally corrugated surface of the metal layer. The nature of these plasmons is a bit different from the plasmons observed on smooth surfaces as they are not propagating, but localized in the small area of confinement the nanostructure offers them. Rough metal surface was simulated using interlayers of $\mathrm{CaF}_{2}$ of varying thicknesses, which provides surfaces of well-defined properties $[18,19]$. Decreased phase velocity of surface plasmons calculated from this simulated interface is a consequence of multiple scatterings on the rough surface (scattering of the plasmon in new direction followed by rescattering into the original direction of the plasmon leading to reduction of the phase velocity at the preserved direction). Damping of the surface plasmons is determined by light emission into the air space. Experiments with monochromatic laser light at $1060 \mathrm{~nm}$ wavelength directed towards rough metal surfaces at $45^{\circ}$ show that in the spectrum of scattered light of reflected beam, the signal for second harmonic of the incoming light $(530 \mathrm{~nm})$ is greatly amplified compared to the reflection on smooth metal surfaces [20]. This is caused by strong enhancement of the local electromagnetic field in the metal in resonance with the incident radiation. This effect has been used in development of the SERS method; it has been confirmed that the enhancement of the Raman signal is mainly due to an electromagnetic effect rather than chemical interaction between the adsorbed molecule and the metal. Since the discovery of this effect in 1970s, strongly enhanced Raman signals were verified for many different molecules attached to various rough metal surfaces (Figure 3 ). The estimated enhancement factors of the Raman signal range from modest factors of $10^{3}-10^{5}$ to enhancement factors of about $10^{10}-10^{11}$ for dye molecules in surface-enhanced resonance Raman spectroscopy (SERRS) experiments. Although singlemolecule capabilities open up exciting perspectives for SERS as a tool in laboratory medicine and for basic research in biophysics, some of the experimental observations still create some controversy and are not yet completely understood. Together with the limitations attributed to the fact that the target molecules have to be attached to SERS-active substrates such as nanometer-sized silver or gold structures, it results in SERS not being a widely spread sensing method yet. In spite of these problems, this technique remains in focus of many research groups $[9,21-23]$.

2.3. Metal Nanoparticles and the Theory of Mie. The properties of localized surface plasmons have been studied for a long time, because the properties of nanostructured metal surfaces on insulating substrates and metal nanoparticles and colloid solutions are visibly influenced by the localized surface plasmon resonance effects. Already in 1908 Mie recognized that submicroscopical gold spheres differ in their optical properties from gold in the form of atoms and that it would be possible to study the optical absorption of colloid solutions to investigate how gold particles are composed. The theory Mie built upon the basis of Maxwell's equations is nowadays used to account for the frequency-dependent response of spherical metal particles to an electric field. According to this theory, the response is governed by real and imaginary parts of the dielectric function. Band positions, widths, and heights of plasmon oscillations, described in this theory as standing waves, depend sensitively on the dielectric function and on the particle radius [24]. Other factors influencing the surface plasmons in metal clusters are composition and shape [25]. Metal clusters with size above a critical value (usually about $5 \mathrm{~nm}$ radius) exhibit redshift of the surface plasmon resonance. This shift increases with increasing size of the particle. Very small particles lose the metallic character, and their plasmons are suppressed. 

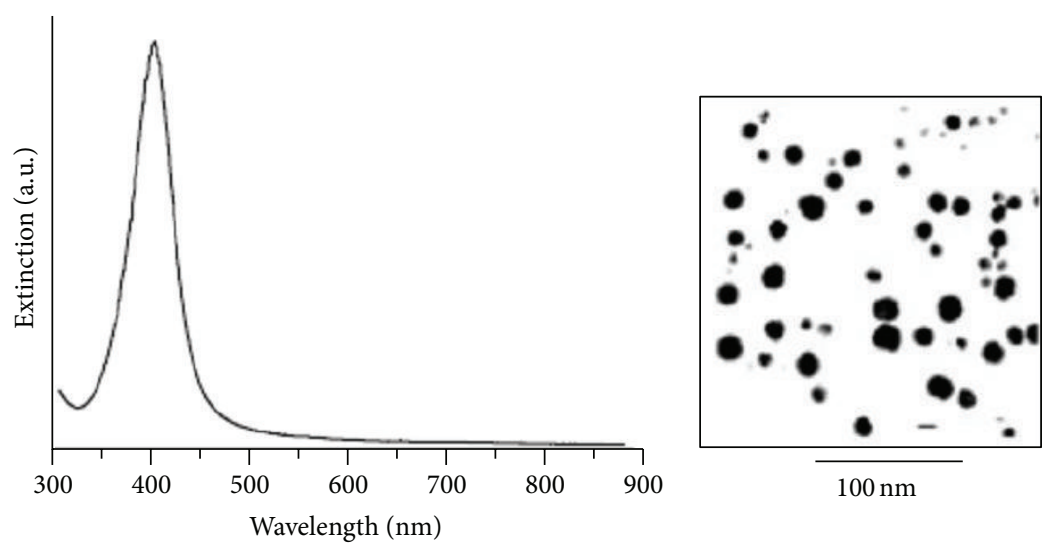

(a)
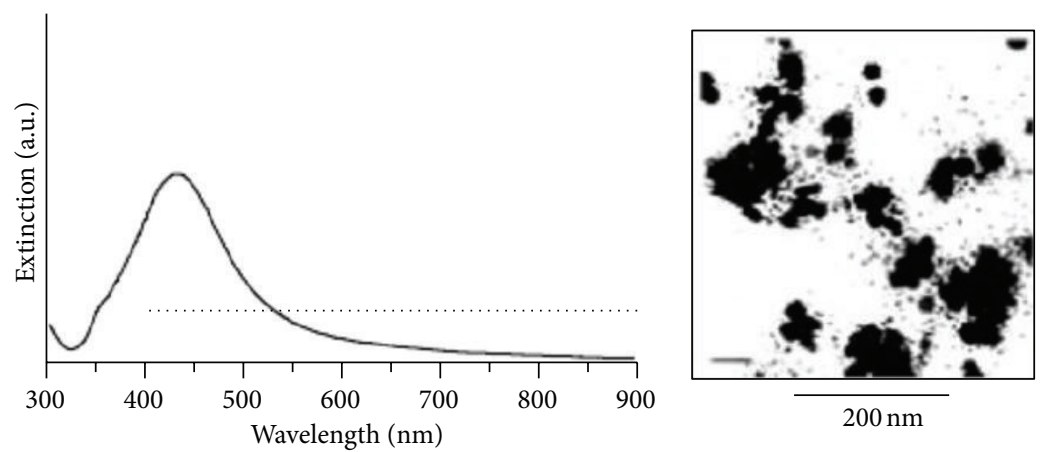

(b)
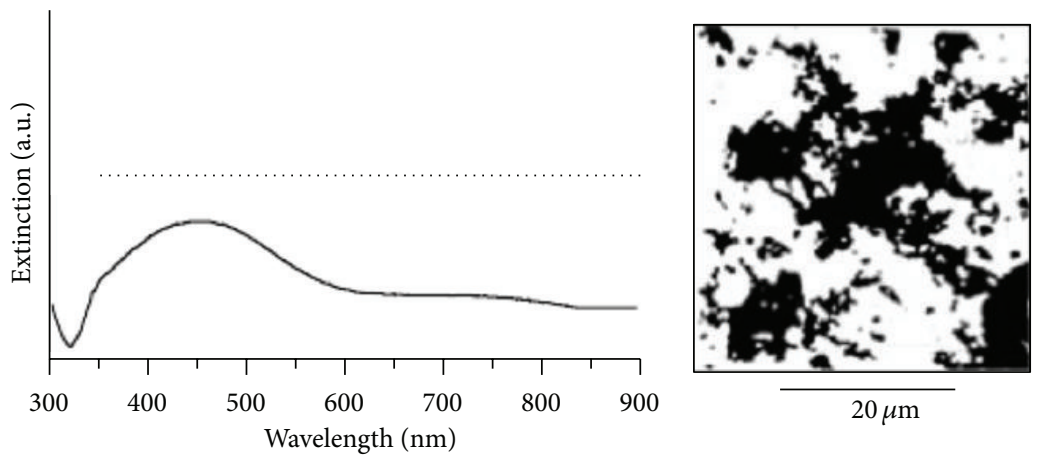

(c)

FIGURE 3: SERS-active colloidal silver particles in different aggregation stages, demonstrating the fractal nature of these structures together with the appropriate extinction curves [9].

For example, the surface plasmon resonance wavelength of gold nanospheres can be tuned over $60 \mathrm{~nm}$ range by varying particle size between 10 and $100 \mathrm{~nm}$ [26]. This is extremely useful for optimization of surface enhanced-effects thermal treatments and also for maximization of the biosensing response of the nanostructures.

The red-shift of the surface plasmon resonance peak induced by a refractive index increase around metal nanostructures is the basis of the simplest sensing application of localized surface plasmons-the refractive index sensor. Noble metal nanoparticles and nanoobjects of various shapes immobilized on a smooth substrate surface are usually used for this purpose. Because localized surface plasmon resonance (LSPR) sensing is based on spectral peak shift, the precision that can be achieved with respect to changes in the refractive index depends on the sensitivity and the peak line width. Larger nanostructures tend to have high sensitivities, but their peaks are broadened by multipolar excitations and radiative damping. The LSPR shift is not strictly linear with the refractive index; therefore narrow spectral range of visible frequencies is usually probed in practice, where linearity of the LSPR shift with refractive index can be approximated. While LSPR sensors can detect changes in bulk refractive index, they are also capable of sensing localized to nanoscale distances from the nanostructure, because field enhancements due to LSPR decay rapidly with 


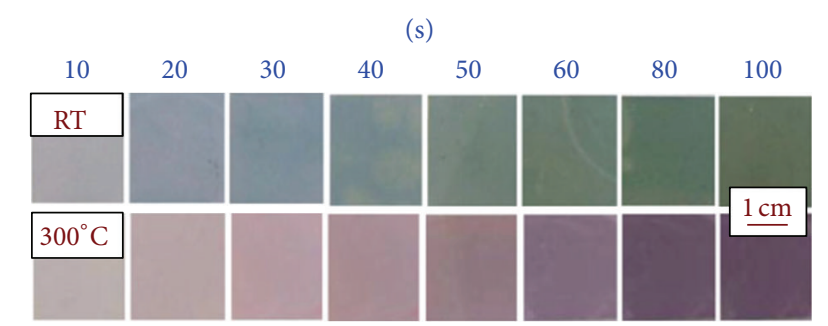

FIGURE 4: Photographs of the glass samples with gold structures sputtered for increasing times. The as-sputtered (RT) and annealed samples $\left(300^{\circ} \mathrm{C}\right)$ are shown [10].

distance from the nanoparticle surface due to strong damping effects. This enables the observation of molecular interactions in the imminent vicinity of the nanoparticle surface $[3,27]$.

Evolution of the character of the material from molecular properties to metallic behaviour has been studied on visible range absorption spectra of clusters of sodium of different size. The atomic/molecular spectra observed at singleatom and 3-atom clusters change for 8-atom cluster in a smooth spectrum with a single absorption band, which shifts with further increasing size of the clusters to the longer wavelengths. Smooth thin films of sodium show monotonic spectra with no bands typical for the curved metal surfaces [28].

Besides the effect of the nanoparticle size, properties of the metal clusters are strongly influenced by their shape and structure as well. There has been a great deal of interest in shapes of nanoparticles that have sharp features or tips, which have been developed through both bottom-up and top-down methods. These include mainly silver and gold nanocubes, gold nanostars, silver nanotriangles, gold bipyramids and gold nanocrescents. One effect of the sharp tips is to produce a red-shift in the plasmon resonance, increasing the refractive index sensitivity [29-37].

\section{Thin Films and 2D Structures}

A great basic example of dependence of optical properties of thin metal films on their thickness and structure is the case of Au nanolayers. Au is a good material for demonstration of the processes that take place during nanostructuring because of its great chemical stability. Švorčík et al. in [10] employed various analytical methods to determine the structure, thickness, morphology, and other properties of sputtered Au films. Annealing of the sputtered films at temperatures as low as $300^{\circ} \mathrm{C}$ leads to coalescence of the material and formation of nanoisland-like structure, which can be attributed to decrease of melting temperature of gold in the form of very thin film. The high surface energy between gold and glass substrate then leads to formation of discontinuous structure of particles spread quite evenly over the substrate surface, whose size depends on the thickness of the deposited Au layer prior to the annealing process.

The color of the continuous sputtered thin Au films ranges from blue to green with increasing thickness turning to the yellow color of the bulk gold for layers of thickness above $30 \mathrm{~nm}$. The discontinuous layers of Au prepared by postdeposition annealing turn in color to the red shade ranging from light pink of the thinnest layers to dark violet for longer sputtering times (Figure 4). This effect is well described from the comparison of UV-Vis spectra and surface morphology of the samples. As annealing of the Au film leads to the formation of island-like structure (Figure 6), surface plasmons in the material become localized, and SPR band arises. While SPR band appears only for very short deposition times for unannealed samples, annealed samples of sputtered gold still show SPR band for layers of effective thickness of about $35 \mathrm{~nm}$. The SPR band of the annealed samples diminishes then for the samples with thickness above $40 \mathrm{~nm}$, where the gold islands become too big to support localization and excitation of surface plasmons (Figure 5). Thus by this simple method based on the control of the Au film thickness prior to annealing, the nanostructured materials with wide variety of different optical absorption spectra in the visible region are produced.

The ellipsometric measurements of the sputtered thin Au films in [38] were used to determine refractive index and to calculate dielectric function. The real part of the dielectric function shows change from the shape typical for semiconductors and insulators to that typical for metals with increasing time of deposition. Annealing leads to occurrence of this transition at much longer deposition times. This measurement may raise some controversy about whether it is possible to calculate optical band gap from the absorption spectrum of the thin Au film. Tauc's method of optical band gap calculation has been used to calculate band gaps in semiconducting materials [39]. According to the ellipsometric measurements, the thin Au island-like film may possess semiconducting properties. The absorption spectrum of these systems is, however, strongly influenced by the SPR absorption band, which can make it difficult to apply this method of band gap calculation.

A different approach for the formation of nanostructured $2 \mathrm{D}$ assemblies of noble metals is to bind a pre-prepared colloid solution of nanoparticles to a beforehand treated substrate. Glass as the usual go-to choice for the substrate has a low affinity to noble metals. It is therefore necessary to modify its surface to make it possible to bind nanoparticles to it. For this purpose, silanization of the glass with various organic substances is often employed. In [11] glass plates were treated with aminopropyltrimethoxysilane and subsequently treated with colloid solutions of Au. UV-Vis spectra in this case showed strong dependence of the absorption on the time of the sample treatment in the colloid solution (Figure 7). Spectra with distinct SPR absorption bands were obtained, which indicates a successful binding of the nanoparticles. In [40] a similar method was used to compare the effectiveness of surface modification of the glass substrates by aminopropyltrimethoxysilane and mercaptopropyltrimethoxysilane. Viability of Au nanoparticles prepared by different methods (laser ablated, citrate and borohydride reduced) was also studied. Notably, it was found that aminopropyltrimethoxysilane is better for achieving silanization of the glass than mercaptopropyltrimethoxysilane, which is in contradiction to the usually accepted opinion that there 


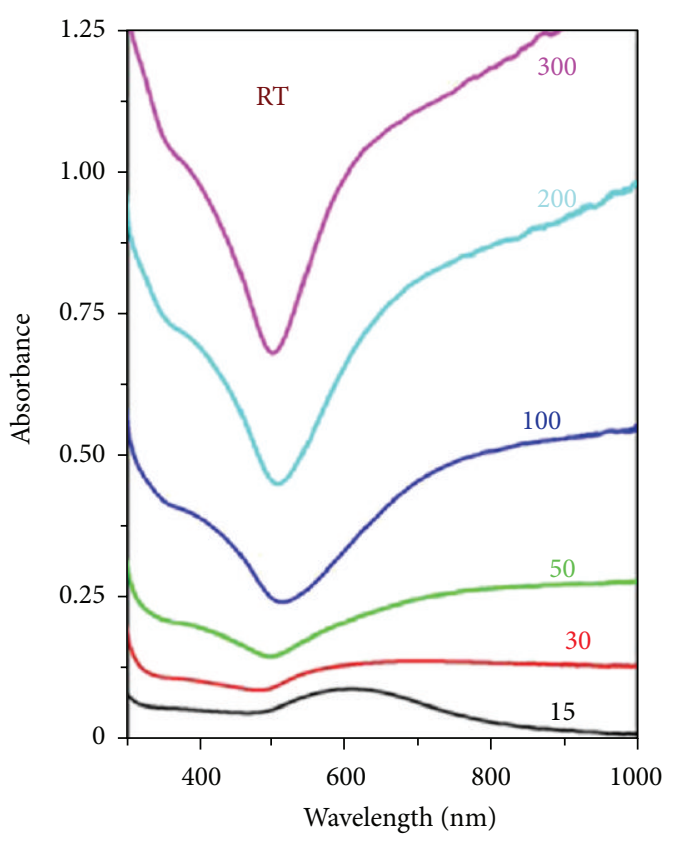

(a)

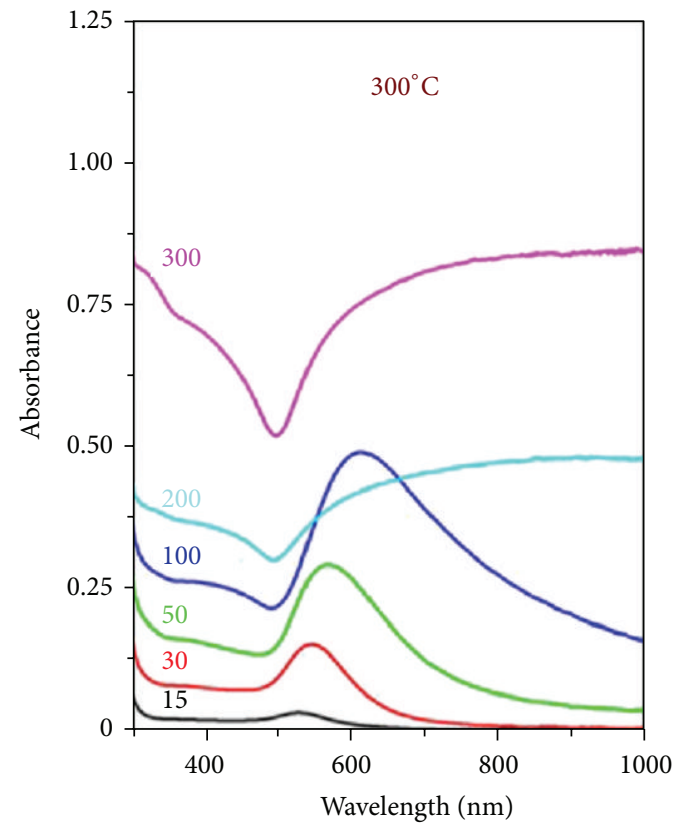

(b)

FIGURE 5: UV-Vis spectra of gold structures sputtered on glass before $(\mathrm{RT})$ and after annealing $\left(300^{\circ} \mathrm{C}\right)$. The numbers of lines refer to sputtering times in $s[10]$.

is very strong interaction between noble metals and-SH groups. This effect can be, however, influenced by the type of used nanoparticle stabilization, because the nanoparticles prepared by laser ablation without any stabilizing agents were found to be unsuitable for the routine preparation of the samples. The structures prepared in this way were tested for their usability as a SERS substrate. Another example of employment of silanized glass substrates is in [12]. Subsequent modification of the immobilized nanoparticles by selfassembled monolayers of mercaptopropionic acid was used to bind biotin and this structure was used as a detection device for interrogation of interactions with fibrinogen. A significant change in the absorption spectrum maximum was observed after the introduction of fibrinogen (Figure 8). A different approach for employing self-assembled monolayers for Au nanoparticle immobilization was studied in [41]. Flat gold substrate surface was modified by 1,10 -decanedithiol, which formed structures with one-SH group bound to the surface and the other prepared to bind the subsequently introduced Au nanoparticle. A process of aggregation leading to structure of multiple layers of nanoparticles bound to the modified surface was observed. The obtained structures were tested for SPR detection of bovine serum albumin.

Examples of possible applications of such structured noble metal films are as was mentioned before in various analytical methods (particularly SERS), but also an attractive method to detect organic gases directly has been suggested in [42], where the dependence of SPR band position and shape on surrounding media refractive index has been employed using polymer overlayer able to absorb organic vapours. Shifts of SPR band have been also studied after direct absorption of organic gases on structured Au and Ag surfaces
[43]. Another interesting example of possible application of thin Au films is in enhancing of organic solar cells, where both optical and electrical properties of the material are very important [44].

\section{Nanoparticles}

The ability to attach noble metal nanoparticles to surfaces may be very useful, especially if we realize that the prepared island-like 2D structure retains the properties of the original nanoparticle solution. If we were able to bind nanoparticles of various shapes, sizes, structure, and composition to solid surfaces without changing their character in the process, we could profit from advantages of both the specific particles and the $2 \mathrm{D}$ noble metal assembly. In this review, we will only mention several of the interesting recent observations concerning optical properties of noble metal nanoparticles, because there are exhaustive recent reviews focusing just on noble metal nanoparticles preparation and properties $[26,45$, 46].

Optical properties of metal nanoparticles are strongly dependent on their shape. Many recent developments have been made in regard to the control of the nanoparticle growth. In [47] properties and possible applications of various shapes (spheres, cubes, rods, and wires) of Au and Ag are discussed. Methods of preparation and shape control of gold nanoparticles with interesting comparison of gold nanoframes SPR absorption dependence on different solvents can be found in [13].

Measurement of optical absorption of colloid solutions may be used in the reverse sense as well-to determine concentration, size, and distribution of nanoparticles in the 

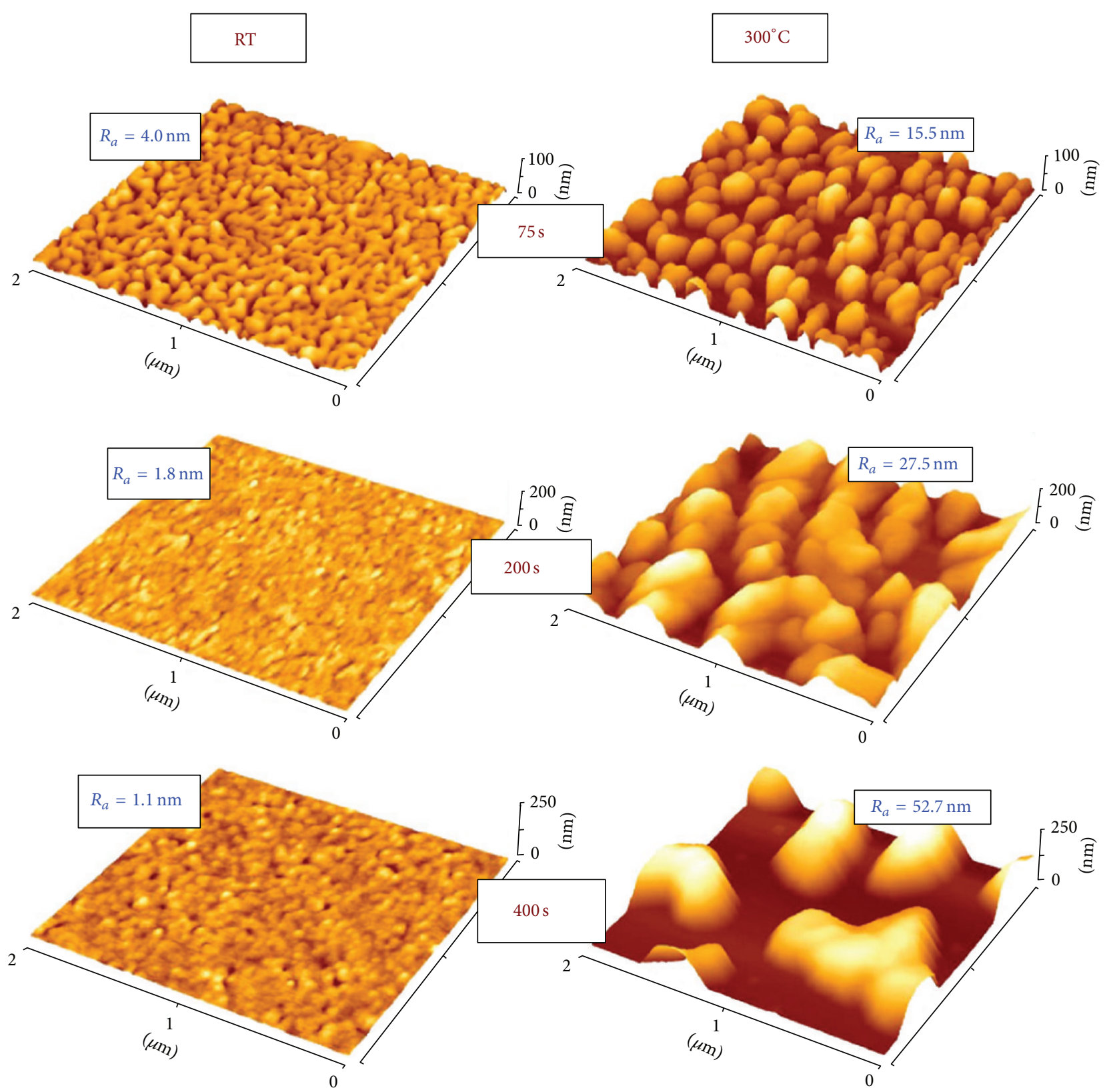

FIGURE 6: AFM images of gold structures sputtered for 75,200 , and $400 \mathrm{~s}$ on glass substrate before (RT) and after annealing ( $\left.300^{\circ} \mathrm{C}\right) . R_{a}$ is the average surface roughness in $\mathrm{nm}[10]$.

colloid solution. Correlation of width of the SPR peak of Au nanoparticles with their size and distribution was used to determine properties of colloid solutions in [48]. Interesting dependence of SPR absorption on measurement temperature is discussed as well. In a more recent study, mean free path correction was applied to classical Mie theory to characterize solutions of $\mathrm{Au}$ nanoparticles of size in the range of $5 \mathrm{~nm}$ to $100 \mathrm{~nm}$. Equations as well as tables for particle size and concentration determination were supported [49].

An attractive method of large-scale preparation of monodisperse colloid solutions of very small Ag nanoparticles is discussed in [50]. In the synthesis of ultra-small Ag nanoparticles, oleylamine was used both as the reducing agent and surfactant, and oleic acid as the cosurfactant and cosolvent. $\mathrm{Ag}$ nanoparticles as small as $1.7 \mathrm{~nm}$ were synthesized by controlling the growth by changing the heating rate. UVVis spectra were measured for samples of solutions after different times of the synthesis to evaluate the state of the process (Figure 9). The measurements show an interesting progress in the nature of the spectra. During the last 2 min of the reaction time, a strong peak at $430 \mathrm{~nm}$ appears and grows rapidly, which is attributed to plasmonic scattering of Ag nanoparticles. This means that nucleation and growth took place in a very short period of time, less than 2 min at $180^{\circ} \mathrm{C}$. In the controlled experiment, where oleic acid was not added to the reaction mixture, it was observed that 


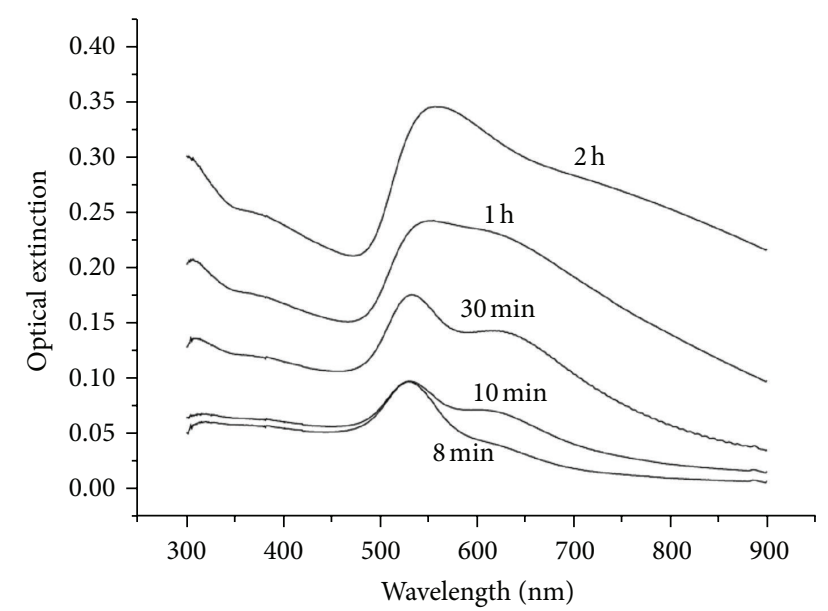

FIGURE 7: Effect of soaking time in the gold colloidal suspension on the UV absorbance of gold-decorated silanized glass substrates. Initial aminopropyltrimethoxysilane concentration was 5\% [11].

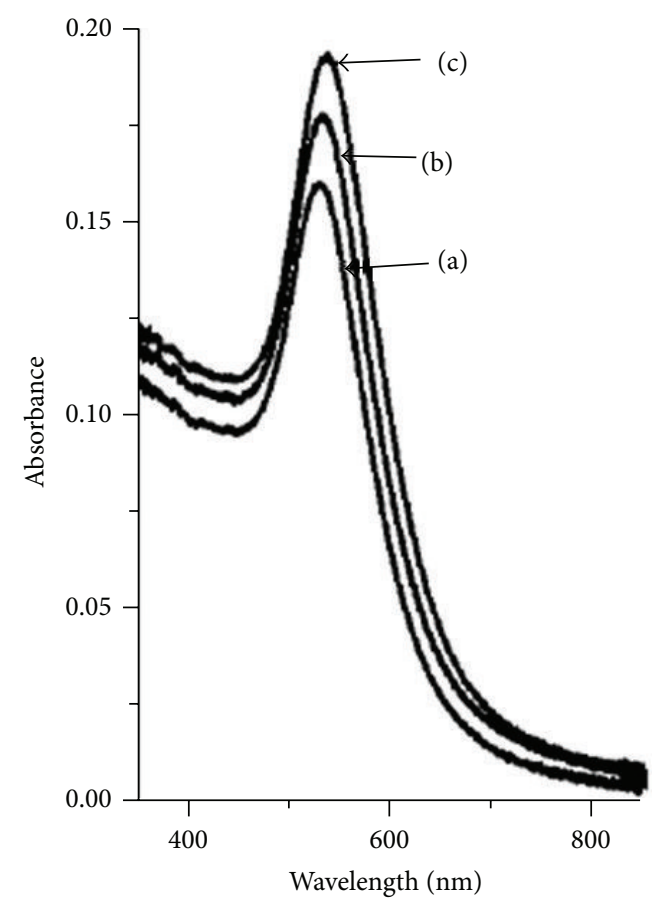

FIGURE 8: Absorbance spectrum of a mercaptopropionic acid functionalized colloidal gold monolayer on glass (a). Change in spectrum after 2 hours of incubation in (b) 10 and (c) $100 \mu \mathrm{g} \cdot \mathrm{mL}$ solution of fibrinogen [12].

the nanoparticles were formed at temperatures much lower, yielding only polydisperse silver particles. It seems that the coordination of oleic acid rather than oleylamine stabilizes the Ag monomers and enables burst of nucleation for the formation of uniform ultra-small nanoparticles.

Another interesting method of Ag nanoparticles preparation uses chitosan as a reduction agent in a one-step preparation process to achieve a more environmentally friendly procedure [51]. Nanoparticles prepared by similar two-step process were studied in regard to their antibacterial activity [14]. UV-Vis extinction spectra in this case document the successful synthesis of silver nanoparticles (Figure 10). The spectra exhibit four characteristic peaks corresponding to different modes of plasmon excitation. These bands correspond to the formation of triangular Ag nanoplates.

$\mathrm{Au}$ nanoparticles are often used in biosensing applications. AuNPs are used as carriers for antibodies or other active molecules; methods using aggregation of AuNPs are based on shifts of the SPR absorption band with formation of AuNP clusters; nanoparticles are used to enhance various luminescence effects [52]. Recently, the specific ability of the particles to enhance electrochemiluminescence of peroxydisulfate used in constructing of biosensor has been reported [53].

\section{Bimetallic Particles and Layers}

We have already discussed how properties of materials change, when size of their structural elements decreases to nanoscopic scale without change of the composition of the material itself. Material properties are affected in a desirable way just by modification of basic structure features. This process is similar to that in which we create mixes of different compounds-by changing the ratio and distribution of the used materials we can tune the properties of the resulting structure. These two procedures can be combined, when we use two different metals to form a nanostructure or nanoparticle (Figure 11).

Bimetallic core-shell Au/Ag nanoparticles can be prepared by two-step subsequent reduction of two metal salts in solution or by electroless deposition of silver onto gold nanoparticle cores. The formation of the bimetallic interface was found to increase sensitivity of LSPR sensors based on this structure [54]. Preparation of the core-shell nanoparticles in the reverse order than in the previous work $(\mathrm{Ag} / \mathrm{Au}$ in this case) was accomplished by deposition of $\mathrm{Au}$ onto previously prepared Ag cores. Significant color change from yellow-amber to dark-amber to grey to grey-purple and finally to purple was observed during the Au deposition (Figure 12). Great attention was paid to the shift of the SPR peak position of the forming particles in regard to the amount of the Au introduced [55]. Absorption spectrum of alloy nanoparticles prepared by laser irradiation of mixture of colloidal solutions of Ag and Au nanoparticles (prepared beforehand by laser ablation in liquid environment) was found not to be a simple combination of the $\mathrm{Au}$ and $\mathrm{Ag}$ colloid solution spectra (Figure 13). Also, the dependence of the resulting spectra on the wavelength of the laser used for the preparation of the alloyed nanoparticles was observed [56]. A summary of preparation methods and means to achieve monodisperse colloid solutions of $\mathrm{Au} / \mathrm{Ag}$ bimetallic nanoparticles was recently published [57].

An example of specific application of optical properties of thin noble metal films is alloyed Pd hydrogen sensor. The intensity of light reflected from thin Pd film was found to be strongly dependent on the $\mathrm{H}_{2}$ absorbed in the layer of the sensor in SPR arrangement [58]. The Pd/Ni alloy was 
$180^{\circ} \mathrm{C} 180^{\circ} \mathrm{C} 180^{\circ} \mathrm{C}$

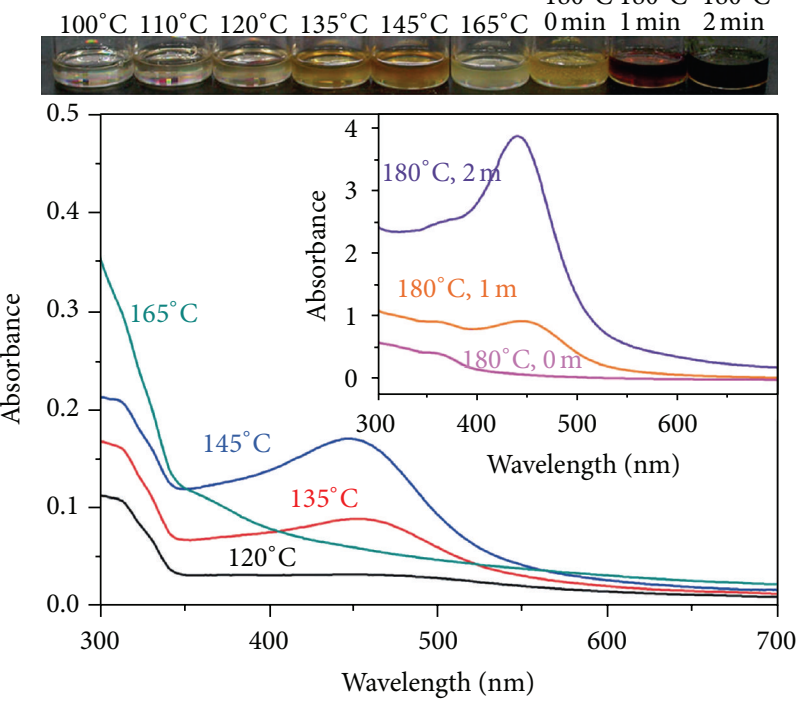

(a)

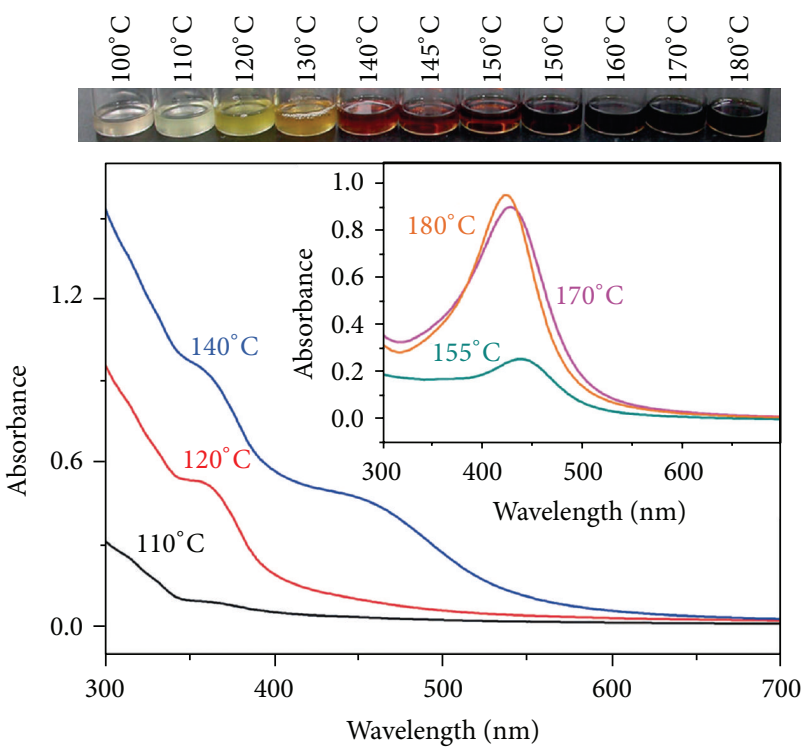

(b)

FIGURE 9: UV/Vis absorption spectra of Ag nanoparticles in various stages of preparation together with corresponding solution photographs. Reaction heating rate was (a) $10^{\circ} \mathrm{C} \cdot \mathrm{min}^{-1}$ and (b) $1^{\circ} \mathrm{C} \cdot \mathrm{min}^{-1}[13]$.

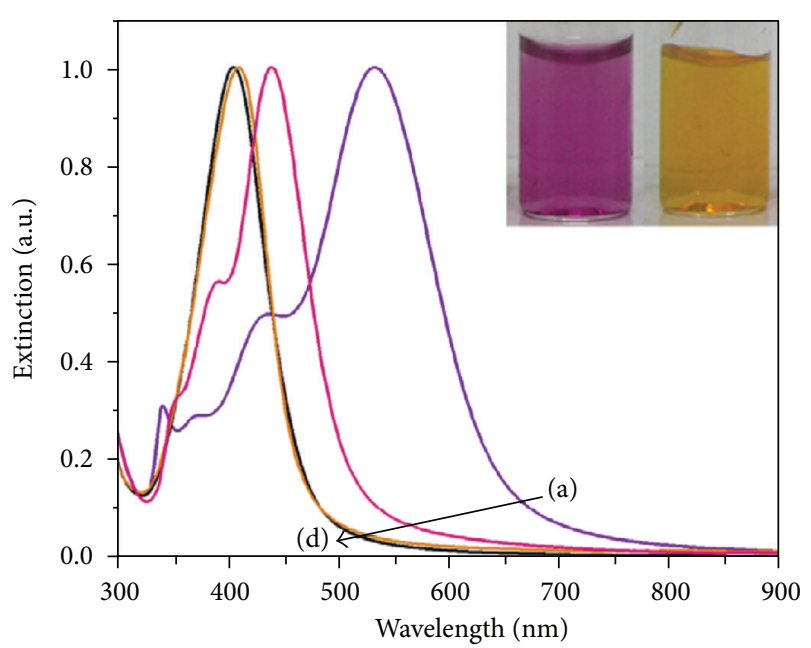

FIGURE 10: Normalized UV-Vis extinction spectra of silver nanoparticles enveloped in chitosan synthesized at $35^{\circ} \mathrm{C}$ with $16.5 \mathrm{mM}$ trisodium citrate concentration (a) and with $4 \mathrm{mM}$ trisodium citrate concentration (b) and at $0^{\circ} \mathrm{C}(\mathrm{c})$, respectively. A reference sample without chitosan at $0^{\circ} \mathrm{C}$ is shown in (d). The photographs correspond to silver colloids prepared at $35^{\circ} \mathrm{C}$ (left) and $0^{\circ} \mathrm{C}$ (right) [14].

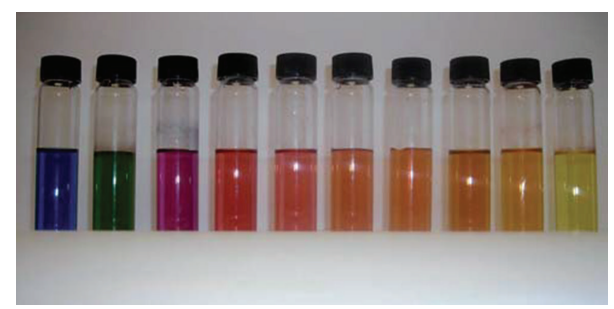

Figure 11: Colloidal solutions of $\mathrm{Ag}$, $\mathrm{Au}$. and $\mathrm{Ag}-\mathrm{Au}$ bimetallic nanoparticles [15]. found to be far more suited than pure palladium for the use in the sensor, owing to its greater mechanical strength and resistance to poisoning by other chemical species. Later, $\mathrm{Au}$ was introduced in the hydrogen sensing alloy, which can prevent certain phase transitions in the Pd layer and lead to better sensitivity of the resulting device [59].

\section{Metal Structures Encapsulated in Semiconductors and Dielectrics}

The dependence of specific optical absorption of nanostructured interfaces on the refractive index of surrounding medium brought to attention a possibility to use certain transparent solids to encapsulate the noble metal structures and improve their properties in this way. Therefore, nanocomposite materials consisted of noble metal thin layer structures encapsulated in semiconductors or dielectrics, or nanoparticles dispersed in such medium were developed. As with thin layers deposited on solid substrates, optical absorption of these composite materials can be modified by annealing. In this case, the annealing temperatures must be quite higher to achieve similar results, because the structure is stabilized by the surrounding solid [60].

The annealing temperature dependence of SPR peak in the UV-Vis spectra was studied in the case of Au structures encapsulated in $\mathrm{TiO}_{2}$ (Figure 14). With increasing temperature of annealing, strong SPR peak of the nanostructured $\mathrm{Au}$ arises. The changes in the absorbance spectra can be detected already at $300^{\circ} \mathrm{C}$, also confirming the first crystallization evidences through the $\mathrm{Au}$ clusters precipitation. Important to emphasis is that the SPR activity starts to develop for temperatures above $200^{\circ} \mathrm{C}$ and becomes higher at higher temperatures. When the samples were annealed between 


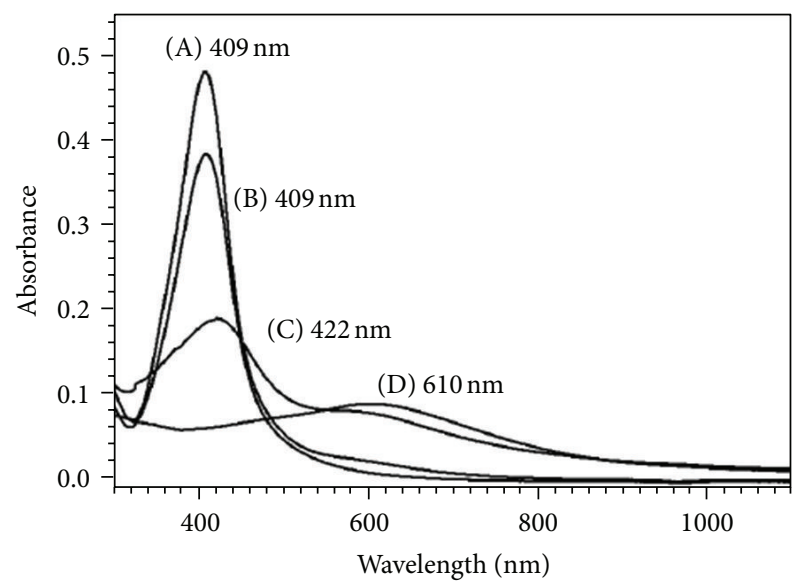

FIGURE 12: UV-Vis spectra for $\mathrm{Ag}$ and $\mathrm{Ag} / \mathrm{Au}$ particles prepared with increasing $\mathrm{Au}$ content, as-synthesized $\mathrm{Ag}$ nanoparticles (A), 5\% $\mathrm{Au}$ (B), $15 \% \mathrm{Au}(\mathrm{C}), 25 \% \mathrm{Au}(\mathrm{D})$ [55].

300 and $500^{\circ} \mathrm{C}$, the optical density increases, together with a slight red-shift. The red-shift of the SPR peak can be attributed to both increasing size of the Au clusters and change in the value of the $\mathrm{TiO}_{2}$ dielectric constant, as different ratios of amorphous $\mathrm{TiO}_{2}$ to anatase are stable at different temperatures [61].

Enhancement of $\mathrm{NiO}$ and $\mathrm{ZnO}$ thin film gas sensing by addition of $\mathrm{Au}$ nanoparticles into the structure was observed. Au nanoparticles embedded into the films by solgel method enabled the use of the structure as an optical sensor for pollutant gases-hydrogen, $\mathrm{CO}$, and $\mathrm{NO}_{2}$. The enhancement of the electrical detection was observed as well as improvement in dynamic characteristics of the sensor [62]. Another interesting structure for gas sensing was developed using $\mathrm{ZnO}$ :Ga nanowire. Adsorbing Au nanoparticles into the wire leads to increase of sensitivity of this sensor to $\mathrm{CO}$ by the order of magnitude [63].

Au nanoparticles were dispersed in $\mathrm{CoO}$ thin film using chemical solution approach combined with spin coating method. Interesting trend of evolution of SPR peak position was observed with increase of nanoparticle concentration (Figure 15). The peaks exhibit a red-shift and intensify with increasing Au content from 10 to $40 \mathrm{~mol} \%$, but show a blue shift and weaken from 40 to $60 \mathrm{~mol} \%$ [64]. An interesting method of Au nanoparticles cultivation in borosilicate glasses of different viscosities was studied in [67]. The particle growth took place at $600^{\circ} \mathrm{C}$. In more viscous glasses very small particles formed, with decreased viscosity, and larger particles showing SPR band appeared. Optical absorption spectra were used to calculate the size of formed nanoparticles and documented great influence of the glass viscosity on the kinetics of the $\mathrm{Au}$ nanoparticle formation process. Another intriguing type of matrix for nanoparticle embedding is polymers. Polytetrafluorethylene, poly(methyl methacrylate), and polyamide 6 were used in [68] as matrices for $\mathrm{Ag}$ nanoclusters. Nanocomposite films were prepared by vapour phase codeposition in high vacuum. The influence of various

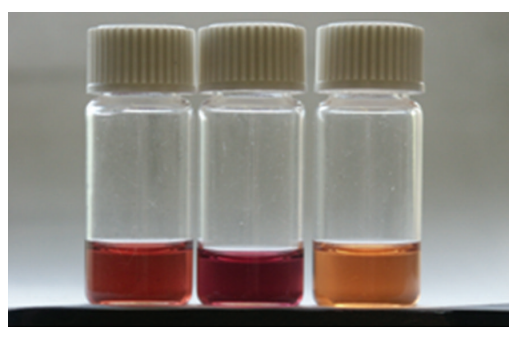

(a)

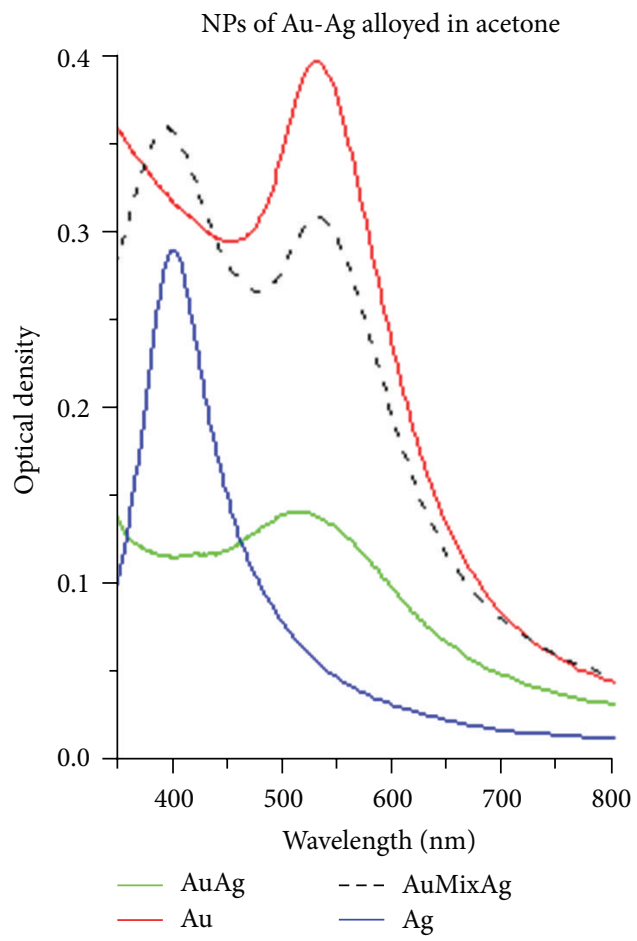

(b)

Figure 13: Photograph of mixture of colloidal solutions (a) of Au and $\mathrm{Ag}$ particles. Initial mixture (left), after 2 hours (middle), and after 4 hours (right) of exposure to $\mathrm{Cu}$ laser radiation. (b) Spectra documenting formation of alloyed Au-Ag particles in acetone. Blue and red lines are the absorption spectra of initial NPs of Ag and Au, respectively. Dashed line is the absorption spectrum of the mixture of individual colloidal solutions prior to laser exposure. The green curve is the absorption spectrum of alloyed Au-AgNPs obtained by laser exposure of the mixture [56].

matrices and Ag clusters concentration on transmission spectrum of the material was tested.

$\mathrm{Au}$ and $\mathrm{Ag}$ particles embedded in various semiconducting or dielectric matrices can be used in construction of nonlinear optic devices [69]. In regard to study of this phenomenon, $\mathrm{Au} / \mathrm{Ag}$ alloyed particles were prepared in the $\mathrm{SiO}_{2}$ matrix [70].

\section{Influence of Substrate on the SPR}

Since plasmons propagate through all substances surrounding the metal nanostructures where they are excited, besides 


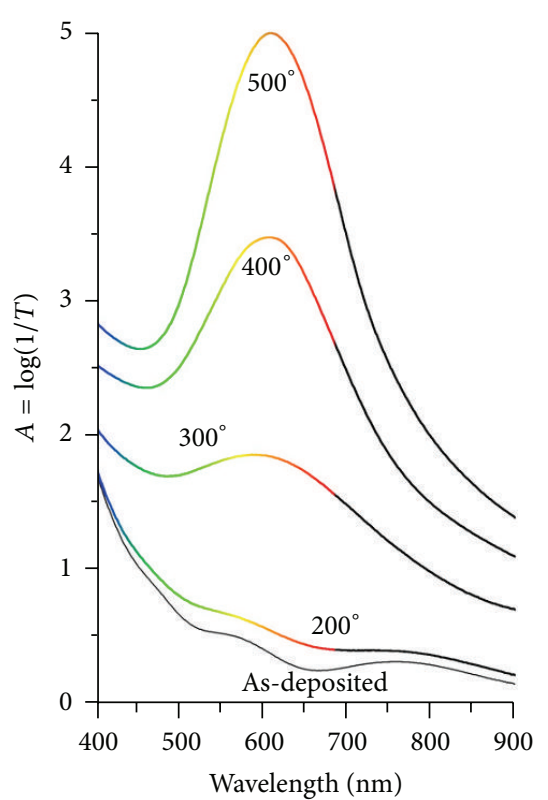

FIgURE 14: Absorbance spectra of $\mathrm{Au}^{\mathrm{TiO}} \mathrm{Ti}_{2}$ at different annealing temperatures [61].

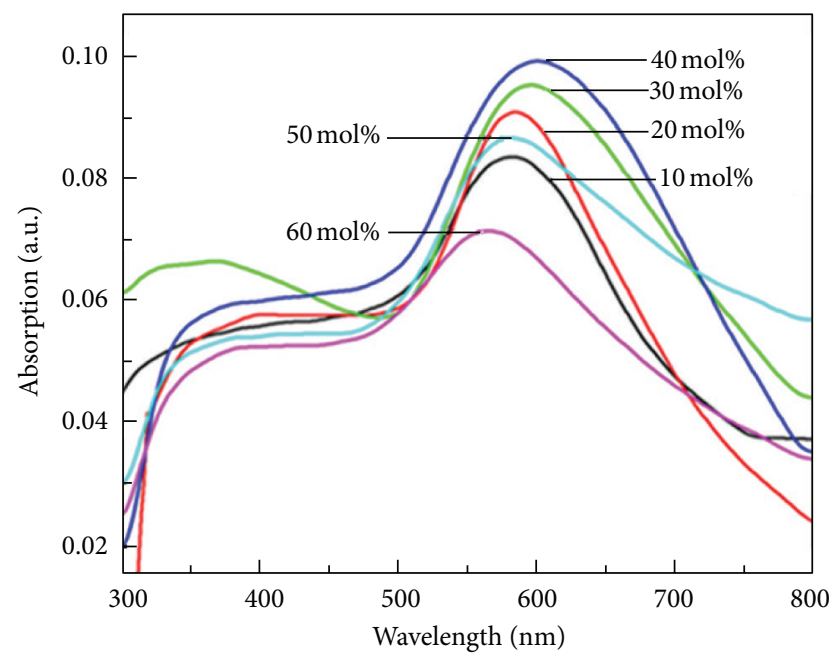

Figure 15: Optical absorption spectra of $\mathrm{Au}: \mathrm{CoO}$ thin films with different contents of Au nanoparticles [64].

properties of overlayers or surrounding atmosphere, the nature of the substrate on which a metal nanolayer is prepared has a crucial effect on the optical properties of the resulting material as well. The choice of a substrate can also have a great influence on the morphology of the growing nanostructure.

The properties of Au thin films deposited on glass and on $\mathrm{SnO} / \mathrm{In}$ layer have been compared in [65]. The suppression of the SPR band of the nanostructured Au in the case of layers prepared on the said semiconducting layer can be seen in transmittance spectra (Figure 16). This effect can be explained by shifts in the stages of the thin film growth depending on the substrate qualities as well as influence of the conductivity and dielectric constant of the substrate on the plasmonic structure of the material.

The effect of the ratio of refractive indexes of overlayer and substrate was studied as well [66]. Nanostructured layers of $\mathrm{Au}$ islands were prepared on substrates of glass, quartz, and indium tin oxide (ITO) and subsequently coated with overlayers of silicon oxide, silicon nitride, ITO, diamond, or amorphous carbonated silicon. Postdeposition annealing was carried out to prepare island-like Au structure. The result was dependent on the quality of substrate with islands being the smallest (about $2 \mathrm{~nm}$ diameter) in the case of ITO and bigger $(4 \mathrm{~nm})$ in the case of glass and quartz (Figure 17).

An interesting type of substrate was proposed in [71], where pores of mesoporous $\mathrm{SiO}_{2}$ were decorated by $\mathrm{Au}$ nanoparticles. An absorption peak at lower wavelength (around $470 \mathrm{~nm}$ ), in addition to the Au SPR peak, induced by ambient aging and subsequent drying at $120^{\circ} \mathrm{C}$ was found. This can be caused by evolution of the structure of $\mathrm{Au}$ particles inside the pores.

Noble metals are well known to function as catalysts of redox reactions in gas sensors. The film of the noble metal has to be porous so that the detected gas could enter the sensitive layer. If we were able to control the structure of the noble metal catalyst film, we could increase the sensitivity of the detector greatly, especially in the low-temperature regime. One of the most studied oxidic semiconducting substrates that enables redox chemical reactions is recently $\mathrm{TiO}_{2}$ as it enables various chemical reactions induced by visible light. The $\mathrm{Au} / \mathrm{Ag}$ nanostructures prepared on its surface can significantly enhance its efficiency by tuning its band gap and mediating the electron transfers. Catalysts based on this principle can be used for degradation of polluting compounds [72], in synthesis of organic substances [73], or for generation of hydrogen for energetic purposes [74].

\section{Conclusions}

The classic assumption that the properties of a material are fully determined by its composition can nowadays be considered completely refuted with the rapid development of the nanotechnology. We can see that the properties of the material of the same composition can be altered in a wide range of values by simply controlling its nanostructure. With decreasing size of the structural elements of the material, quantum effects take place as the energy levels of electrons constituting the material are strongly influenced by the confinement due to the size of a nanoparticle. Confinement in such a way leads to strong intensification of collective oscillations of electrons, known as plasmons, having great effect on electrical and optical properties of the material. The propagating nature of those plasmons means that these properties can be affected by the media surrounding the nanostructure. This effect is a great promise in sensing applications. This also brings many more ways to tune the materials properties, as we can choose different substrates and overlayers for the metal layers, nanostructures, and nanoparticles. Localized plasmons in island-like 2D structures and nanoparticles can be excited simply by incoming 


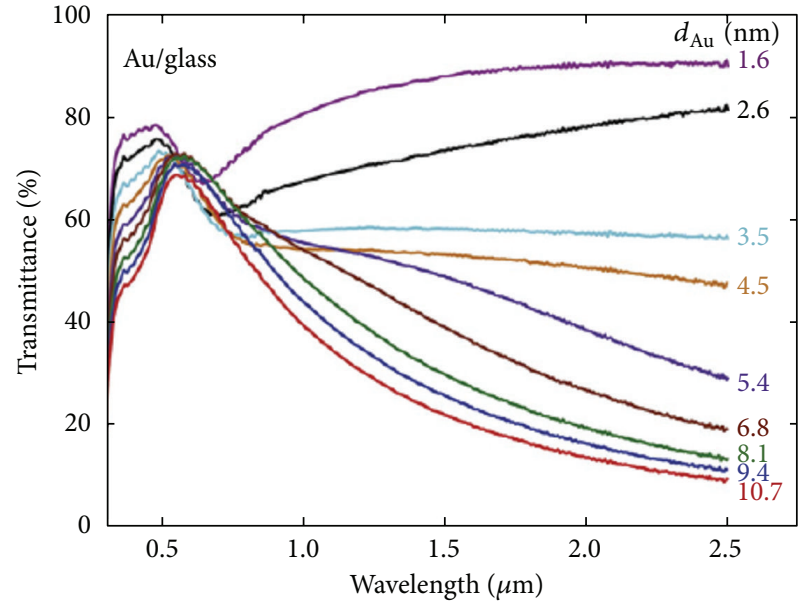

(a)

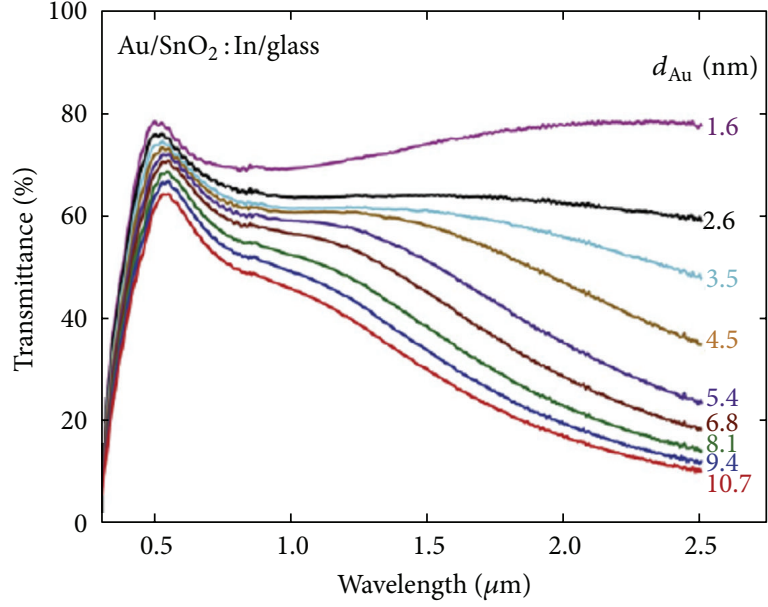

(b)

Figure 16: Spectral transmittance for Au films deposited on glass (a) and on $\mathrm{SnO}_{2}: \mathrm{In}$ (b) [65].
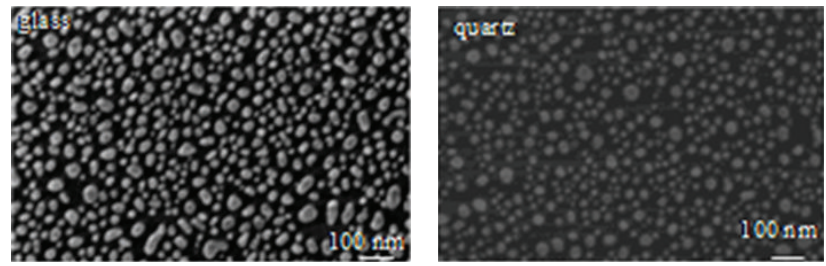

(a)

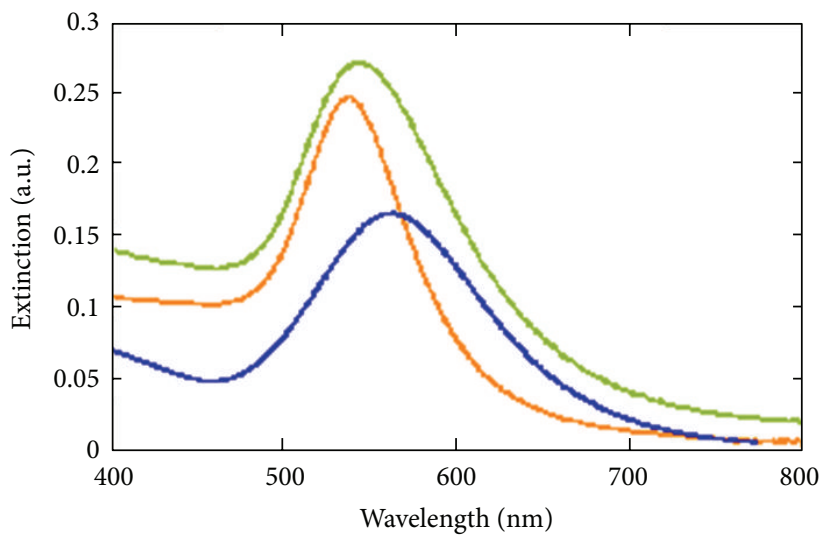

(b)

FIGURE 17: Scanning electron microscopy images (a) of glass/Au, quartz/Au, and ITO/Au nanostructured interfaces formed through thermal deposition of gold film and short high-temperature annealing. Corresponding UV/Vis extinction spectra are shown below (b). The lines in the spectra represent Au nanostructures on glass (green), quartz (red), and ITO (blue) [66].

light and can produce great enhancement of electromagnetic fields, which is studied in regard to improvement of the Raman spectroscopy techniques.

The opportunity to tune properties of materials over a large scale of values opens up brand new possibilities in the applications of prepared structures. The nature of surface plasmons predetermines noble metal nanostructures to be great materials for development of modern label-free sensing methods based on plasmon resonance-SPR and LSPR sensing. The effect of electromagnetic field enhancement of localized plasmons is thoroughly studied by groups specializing in the development of SERS method. Increased reactiveness of noble metal nanoparticles has been found to be of great use in classic methods of gas sensing. Nanostructured noble metals even show great promises in improvement of organic solar cells effectiveness. 


\section{Acknowledgments}

This work was supported by the GACR under Project no. 108/12/G108. The authors thank Mrs. Oliva Kesselová for technical support.

\section{References}

[1] H. Gleiter, "Materials with ultrafine microstructures: retrospectives and perspectives," Nanostructured Materials, vol. 1, no. 1, pp. 1-19, 1992.

[2] E. Roduner, "Size matters: why nanomaterials are different," Chemical Society Reviews, vol. 35, no. 7, pp. 583-592, 2006.

[3] K. M. Mayer and J. H. Hafner, "Localized surface plasmon resonance sensors," Chemical Reviews, vol. 111, no. 6, pp. 38283857, 2011.

[4] A. P. Alivisatos, "Semiconductor clusters, nanocrystals, and quantum dots," Science, vol. 271, no. 5251, pp. 933-937, 1996.

[5] A. D. Yoffe, "Low-dimensional systems: quantum size effects and electronic properties of semiconductor microcrystallites (zero-dimensional systems) and some quasi-two-dimensional systems," Advances in Physics, vol. 42, no. 2, pp. 173-266, 1993.

[6] E. Roduner, Nanoscopic Materials: Size Dependent Phenomena, RSC Publishing, Cambridge, UK, 2006.

[7] H. Raether, Surface Plasmons on Smooth and Rough Surfaces and on Gratings, Springer, New York, NY, USA, 1988.

[8] J. Homola, S. S. Yee, and G. Gauglitz, "Surface plasmon resonance sensors: review," Sensors and Actuators B, vol. 54, no. 1-2, pp. 3-15, 1999.

[9] K. Kneipp, H. Kneipp, I. Itzkan, R. R. Dasari, and M. S. Feld, "Surface-enhanced Raman scattering and biophysics," Journal of Physics Condensed Matter, vol. 14, no. 18, pp. R597-R624, 2002.

[10] V. Švorčík, O. Kvítek, O. Lyutakov, J. Siegel, and Z. Kolská, "Annealing of sputtered gold nano-structures," Applied Physics A, vol. 102, no. 3, pp. 747-751, 2011.

[11] O. Seitz, M. M. Chehimi, E. Cabet-Deliry et al., "Preparation and characterisation of gold nanoparticle assemblies on silanised glass plates," Colloids and Surfaces A, vol. 218, no. 13, pp. 225-239, 2003.

[12] N. Nath and A. Chilkoti, "A colorimetric gold nanoparticle sensor to interrogate biomolecular interactions in real time on a surface," Analytical Chemistry, vol. 74, no. 3, pp. 504-509, 2002.

[13] J. Xiao and L. Qi, "Surfactant-assisted, shape-controlled synthesis of gold nanocrystals," Nanoscale, vol. 3, no. 4, pp. 1383-1396, 2011.

[14] M. Potara, E. Jakab, A. Damert, O. Popescu, V. Canpean, and S. Astilean, "Synergistic antibacterial activity of chitosan-silver nanocomposites on Staphylococcus aureus," Nanotechnology, vol. 22, no. 13, Article ID 135101, 2011.

[15] Q. Zhang, Y. N. Tan, J. Xie, and J. Y. Lee, "Colloidal synthesis of plasmonic metallic nanoparticles," Plasmonics, vol. 4, no. 1, pp. 9-22, 2009.

[16] D. R. Shankaran, K. V. Gobi, and N. Miura, "Recent advancements in surface plasmon resonance immunosensors for detection of small molecules of biomedical, food and environmental interest," Sensors and Actuators B, vol. 121, no. 1, pp. 158-177, 2007.

[17] E. Wijaya, C. Lenaerts, S. Maricot et al., "Surface plasmon resonance-based biosensors: from the development of different
SPR structures to novel surface functionalization strategies," Current Opinion in Solid State and Materials Science, vol. 15, no. 5, pp. 208-224, 2011.

[18] D. Hornauer, H. Kapitza, and H. Raether, "The dispersion relation of surface plasmons on rough surfaces," Journal of Physics D, vol. 7, no. 9, pp. L100-L102, 1974.

[19] A. J. Braundmeier Jr. and E. T. Arakawa, "Effect of surface roughness on surface plasmon resonance absorption," Journal of Physics and Chemistry of Solids, vol. 35, no. 4, pp. 517-520, 1974.

[20] C. K. Chen, A. R. B. de Castro, and Y. R. Shen, "Surfaceenchanced 2nd-harmonic generation," Physical Review Letters, vol. 46, no. 2, pp. 145-148, 1981.

[21] M. Moskovits, "Surface-enhanced spectroscopy," Reviews of Modern Physics, vol. 57, no. 3, pp. 783-826, 1985.

[22] R. A. Tripp, R. A. Dluhy, and Y. Zhao, "Novel nanostructures for SERS biosensing," Nano Today, vol. 3, no. 3-4, pp. 31-37, 2008.

[23] B. Sharma, R. R. Frontiera, A.-I. Henry, E. Ringe, and R. P. Van Duyne, "SERS: materials, applications, and the future," Materials Today, vol. 15, no. 1-2, pp. 16-25, 2012.

[24] G. Mie, "Beitragezur Optik triiber Medien, speziell kolloidaler Metallösungen," Annals of Physics, vol. 25, no. 3, pp. 377-445, 1908.

[25] L. M. Liz-Marzán, "Tailoring surface plasmons through the morphology and assembly of metal nanoparticles," Langmuir, vol. 22, no. 1, pp. 32-41, 2006.

[26] M.-C. Daniel and D. Astruc, "Gold nanoparticles: assembly, supramolecular chemistry, quantum-size-related properties, and applications toward biology, catalysis, and nanotechnology," Chemical Reviews, vol. 104, no. 1, pp. 293-346, 2004.

[27] B. Sepúlveda, P. C. Angelomé, L. M. Lechuga, and L. M. LizMarzán, "LSPR-based nanobiosensors," Nano Today, vol. 4, no. 3, pp. 244-251, 2009.

[28] U. Kreibig and M. Vollmer, Optical Properties of Metal Clusters, Materials Science, Springer, New York, NY, USA, 1995.

[29] L. J. Sherry, S.-H. Chang, G. C. Schatz, R. P. Van Duyne, B. J. Wiley, and Y. Xia, "Localized surface plasmon resonance spectroscopy of single silver nanocubes," Nano Letters, vol. 5, no. 10, pp. 2034-2038, 2005.

[30] H. Chen, X. Kou, Z. Yang, W. Ni, and J. Wang, "Shape- and sizedependent refractive index sensitivity of gold nanoparticles," Langmuir, vol. 24, no. 10, pp. 5233-5237, 2008.

[31] C. L. Nehl, H. Liao, and J. H. Hafner, "Optical properties of starshaped gold nanoparticles," Nano Letters, vol. 6, no. 4, pp. 683688,2006 .

[32] L. J. Sherry, R. Jin, C. A. Mirkin, G. C. Schatz, and R. P. Van Duyne, "Localized surface plasmon resonance spectroscopy of single silver triangular nanoprisms," Nano Letters, vol. 6, no. 9, pp. 2060-2065, 2006.

[33] M. Liu and P. Guyot-Sionnest, "Mechanism of silver(I)-assisted growth of gold nanorods and bipyramids," Journal of Physical Chemistry B, vol. 109, no. 47, pp. 22192-22200, 2005.

[34] K. L. Kelly, E. Coronado, L. L. Zhao, and G. C. Schatz, "The optical properties of metal nanoparticles: the influence of size, shape, and dielectric environment," Journal of Physical Chemistry B, vol. 107, no. 3, pp. 668-677, 2003.

[35] M. Liu, P. Guyot-Sionnest, T.-W. Lee, and S. K. Gray, "Optical properties of rodlike and bipyramidal gold nanoparticles from three-dimensional computations," Physical Review B, vol. 76, no. 23, Article ID 235428, 2007. 
[36] J. M. McMahon, Y. Wang, L. J. Sherry et al., "Correlating the structure, optical spectra, and electrodynamics of single silver nanocubes," Journal of Physical Chemistry C, vol. 113, no. 7, pp. 2731-2735, 2009.

[37] R. Bukasov and J. S. Shumaker-Parry, "Highly tunable infrared extinction properties of gold nanocrescents," Nano Letters, vol. 7, no. 5, pp. 1113-1118, 2007.

[38] V. Švorčík, J. Siegel, P. Šutta et al., "Annealing of gold nanostructures sputtered on glass substrate," Applied Physics A, vol. 102, no. 3, pp. 605-610, 2011.

[39] J. Tauc, Amorphous and Liquid Semiconductors, Springer, New York, NY, USA, 1974.

[40] N. Hajduková, M. Procházka, J. Štěpánek, and M. Špírková, "Chemically reduced and laser-ablated gold nanoparticles immobilized to silanized glass plates: preparation, characterization and SERS spectral testing," Colloids and Surfaces A, vol. 301, no. 1-3, pp. 264-270, 2007.

[41] E. M. S. Azzam, A. Bashir, O. Shekhah et al., "Fabrication of a surface plasmon resonance biosensor based on gold nanoparticles chemisorbed onto a 1,10-decanedithiol self-assembled monolayer," Thin Solid Films, vol. 518, no. 1, pp. 387-391, 2009.

[42] T. Karakouz, A. Vaskevich, and I. Rubinstein, "Polymer-coated gold island films as localized plasmon transducers for gas sensing," Journal of Physical Chemistry B, vol. 112, no. 46, pp. 14530-14538, 2008.

[43] C.-S. Cheng, Y.-Q. Chen, and C.-J. Lu, "Organic vapour sensing using localized surface plasmon resonance spectrum of metallic nanoparticles self assemble monolayer," Talanta, vol. 73, no. 2, pp. 358-365, 2007.

[44] S. D. Yambem, A. Haldar, K.-S. Liao, E. P. Dillon, A. R. Barron, and S. A. Curran, "Optimization of organic solar cells with thin film Au as anode," Solar Energy Materials and Solar Cells, vol. 95, no. 8, pp. 2424-2430, 2011.

[45] T.-H. Tran and T.-D. Nguyen, "Controlled growth of uniform noble metal nanocrystals: aqueous-based synthesis and some applications in biomedicine," Colloids and Surfaces B, vol. 88, no. 1, pp. 1-22, 2011.

[46] E. C. Dreaden, A. M. Alkilany, X. Huang, C. J. Murphy, and M. A. El-Sayed, "The golden age: gold nanoparticles for biomedicine," Chemical Society Reviews, vol. 41, no. 7, pp. 2740 2779, 2012.

[47] A. Seyed-Razavi, I. K. Snook, and A. S. Barnard, "Origin of nanomorphology: does a complete theory of nanoparticle evolution exist?" Journal of Materials Chemistry, vol. 20, no. 3, pp. 416-421, 2010.

[48] S. Link and M. A. El-Sayed, "Size and temperature dependence of the plasmon absorption of colloidal gold nanoparticles," Journal of Physical Chemistry B, vol. 103, no. 21, pp. 4212-4217, 1999.

[49] W. Haiss, N. T. K. Thanh, J. Aveyard, and D. G. Fernig, "Determination of size and concentration of gold nanoparticles from UV-Vis spectra," Analytical Chemistry, vol. 79, no. 11, pp. 4215-4221, 2007.

[50] J. Park, S. G. Kwon, S. W. Jun, B. H. Kim, and T. Hyeon, "Largescale synthesis of ultra-small-sized silver nanoparticles," Journal of Physical Chemistry A, vol. 13, no. 10, pp. 2540-2543, 2012.

[51] A. Murugadoss and A. Chattopadhyay, "A "green" chitosansilver nanoparticle composite as a heterogeneous as well as micro-heterogeneous catalyst," Nanotechnology, vol. 19, no. 1, Article ID 015603, 2008.
[52] X. Cao, Y. Ye, and S. Liu, "Gold nanoparticle-based signal amplification for biosensing," Analytical biochemistry, vol. 417, no. 1, pp. 1-16, 2011.

[53] B. H. Gao, S. N. Ding, O. Kargbo, Y. H. Wang, Y. M. Sun, and S. Cosnier, "Enhanced electrochemiluminescence of peroxydisulfate by electrodeposited Au nanoparticles and its biosensing application via integrating biocatalytic precipitation using selfassembly bi-enzymes," Journal of Electroanalytical Chemistry, vol. 703, pp. 9-13, 2013.

[54] A. Csáki, S. Berg, N. Jahr et al., "Plasmonic nanoparticlesnoble material for sensoric applications," in Gold Nanoparticles: Properties, Characterization and Fabrication, NOVA Science, Huntington, NY, USA, 2009.

[55] D. Mott, N. T. B. Thuy, Y. Aoki, and S. Maenosono, "Synthesis of size and shape controlled silver nanoparticles coated by a thin layer of gold and their use as ultrasensitive biomolecular probes," in Proceedings of the Materials Research Society in Functional Materials and Nanostructures for Chemical and Biochemical Sensing, vol. 1253, pp. 652-663, April 2010.

[56] G. A. Shafeev, "Laser synthesis of gold nanoparticles and the control over their properties," in Gold Nanoparticles: Properties, Characterization and Fabrication, NOVA Science, New York, NY, USA, 2009.

[57] Q. Zhang, J. Xie, Y. Yu, and J. Y. Lee, “Monodispersity control in the synthesis of monometallic and bimetallic quasi-spherical gold and silver nanoparticles," Nanoscale, vol. 2, no. 10, pp. $1962-$ 1975, 2010.

[58] B. Chadwick, J. Tann, M. Brungs, and M. Gal, "A hydrogen sensor based on the optical generation of surface plasmons in a palladium alloy," Sensors and Actuators B, vol. 17, no. 3, pp. 215-220, 1994.

[59] S. Nazarpour, A. Cirera, and M. Varela, "Material properties of Au-Pd thin alloy films," Thin Solid Films, vol. 518, no. 20, pp. 5715-5719, 2010.

[60] L. Armelao, D. Barreca, G. Bottaro et al., "Recent trends on nanocomposites based on $\mathrm{Cu}, \mathrm{Ag}$ and Au clusters: a closer look," Coordination Chemistry Reviews, vol. 250, no. 11-12, pp. 12941314, 2006.

[61] M. Torrell, L. Cunha, A. Cavaleiro, E. Alves, N. P. Barradas, and F. Vaz, "Functional and optical properties of Au:TiO2 nanocomposite films: the influence of thermal annealing," Applied Surface Science, vol. 256, no. 22, pp. 6536-6542, 2010.

[62] E. Della Gaspera, M. Guglielmi, A. Martucci, L. Giancaterini, and C. Cantalini, "Enhanced optical and electrical gas sensing response of sol-gel based $\mathrm{NiO}-\mathrm{Au}$ and $\mathrm{ZnO}-\mathrm{Au}$ nanostructured thin films," Sensors and Actuators B, vol. 164, no. 1, pp. 54-63, 2012.

[63] S.-J. Chang, T.-J. Hsueh, I.-C. Chen, and B.-R. Huang, "Highly sensitive $\mathrm{ZnO}$ nanowire $\mathrm{CO}$ sensors with the adsorption of $\mathrm{Au}$ nanoparticles," Nanotechnology, vol. 19, no. 17, Article ID 175502, 2008.

[64] S.-J. Wang, B.-P. Zhang, L.-P. Yan, and W. Deng, "Microstructure and optical absorption properties of Au-dispersed $\mathrm{CoO}$ thin films," Journal of Alloys and Compounds, vol. 509, no. 19, pp. 5731-5735, 2011.

[65] P. C. Lansåker, K. Gunnarsson, A. Roos, G. A. Niklasson, and C. G. Granqvist, "Au thin films deposited on $\mathrm{SnO}_{2}$ : in and glass: substrate effects on the optical and electrical properties," Thin Solid Films, vol. 519, no. 6, pp. 1930-1933, 2011.

[66] S. Szunerits and R. Boukherroub, "Short and long range sensing on plasmonic nanostructures coated with oxide-based 
dielectrics," in Gold Nanoparticles: Properties, Characterization and Fabrication, NOVA Science, New York, NY, USA, 2009.

[67] J. Vosburgh and R. H. Doremus, "Optical absorption spectra of gold nano-clusters in potassium borosilicate glass," Journal of Non-Crystalline Solids, vol. 349, no. 1-3, pp. 309-314, 2004.

[68] H. Takele, H. Greve, C. Pochstein, V. Zaporojtchenko, and F. Faupel, "Plasmonic properties of Ag nanoclusters in various polymer matrices," Nanotechnology, vol. 17, no. 14, pp. 34993505, 2006.

[69] J. P. Huang and K. W. Yu, "Enhanced nonlinear optical responses of materials: composite effects," Physics Reports, vol. 431, no. 3, pp. 87-172, 2006.

[70] P. Sangpour, O. Akhavan, and A. Z. Moshfegh, "The effect of $\mathrm{Au} / \mathrm{Ag}$ ratios on surface composition and optical properties of co-sputtered alloy nanoparticles in $\mathrm{Au}-\mathrm{Ag}: \mathrm{SiO}_{2}$ thin films," Journal of Alloys and Compounds, vol. 486, no. 1-2, pp. 22-28, 2009.

[71] G. Fu, W. Cai, Y. Gan, and J. Jia, "An ambience-induced optical absorption peak for $\mathrm{Au} / \mathrm{SiO}_{2}$ mesoporous assembly," Chemical Physics Letters, vol. 385, no. 1-2, pp. 15-19, 2004.

[72] D. Gong, W. C. J. Ho, Y. Tang et al., "Silver decorated titanate/titania nanostructures for efficient solar driven photocatalysis," Journal of Solid State Chemistry, vol. 189, pp. 117-122, 2012.

[73] R. Kaur and B. Pal, "Size and shape dependent attachments of $\mathrm{Au}$ nanostructures to $\mathrm{TiO}_{2}$ for optimum reactivity of $\mathrm{Au}-\mathrm{TiO}_{2}$ photocatalysis," Journal of Molecular Catalysis A, vol. 355, pp. 39-43, 2012.

[74] H. Yuzawa, T. Yoshida, and H. Yoshida, "Gold nanoparticles on titanium oxide effective for photocatalytic hydrogen formation under visible light," Applied Catalysis B, vol. 115-116, pp. 294302, 2012. 

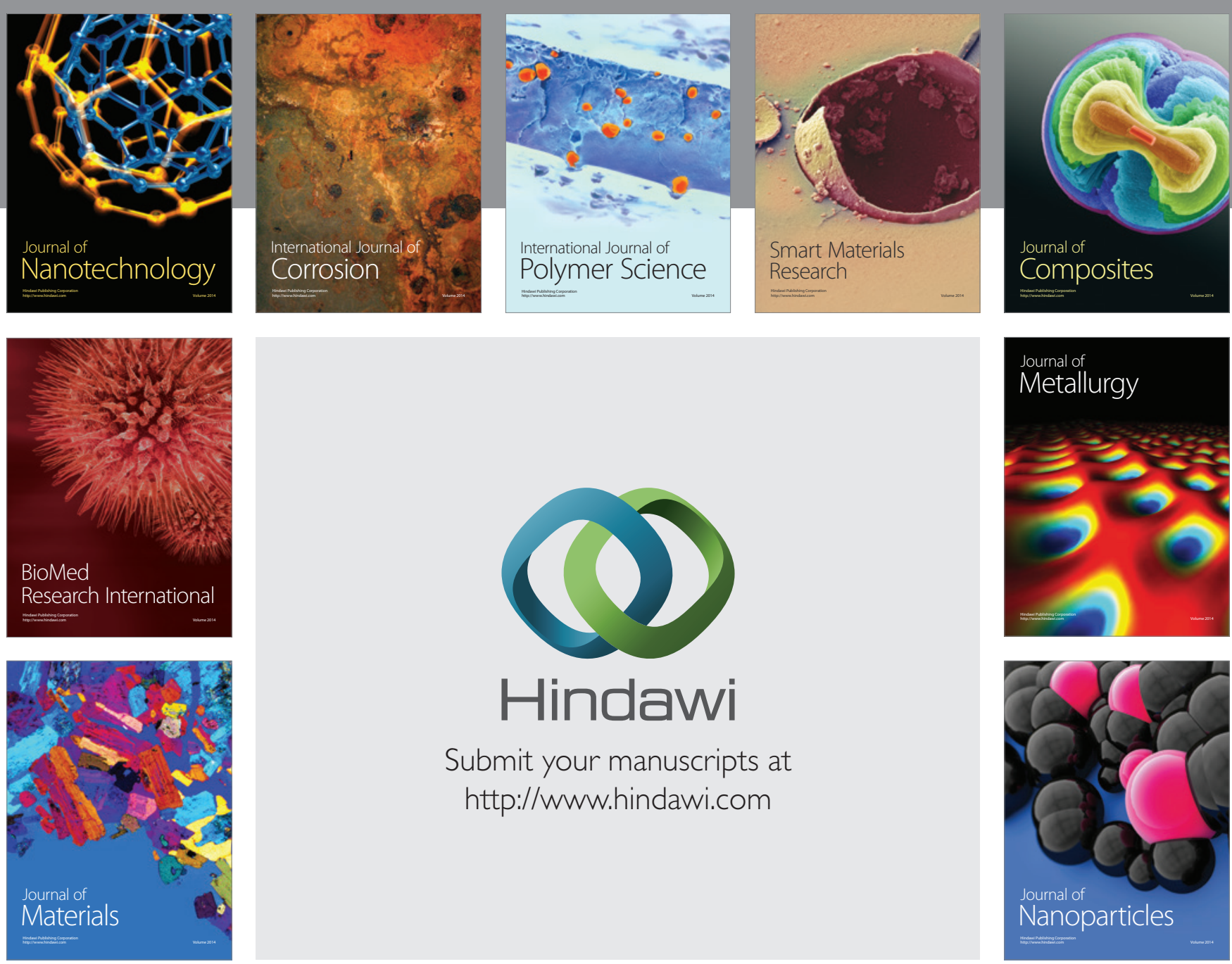

Submit your manuscripts at http://www.hindawi.com
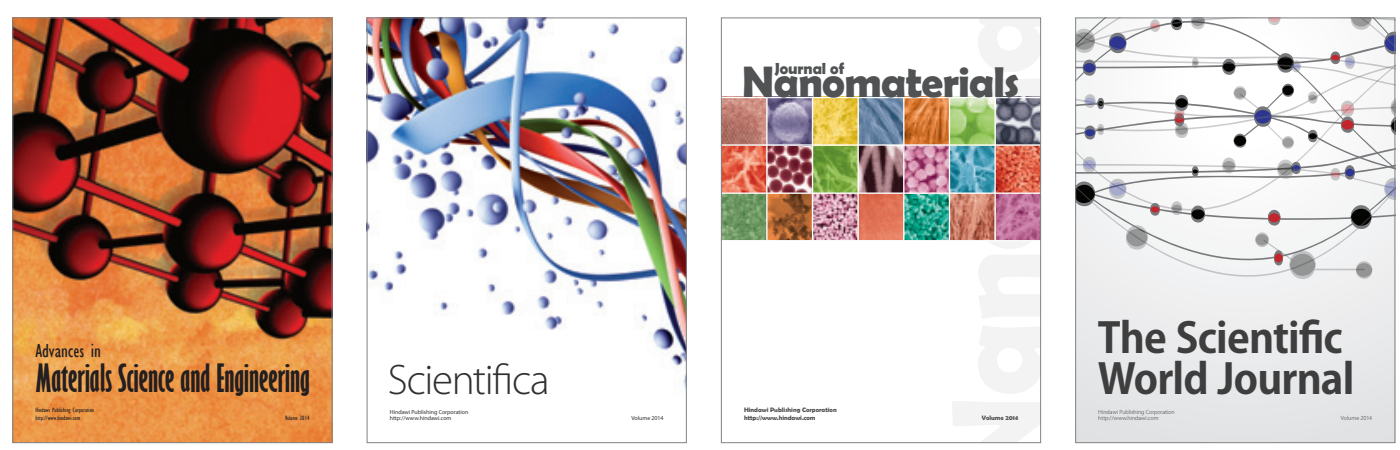

\section{The Scientific World Journal}
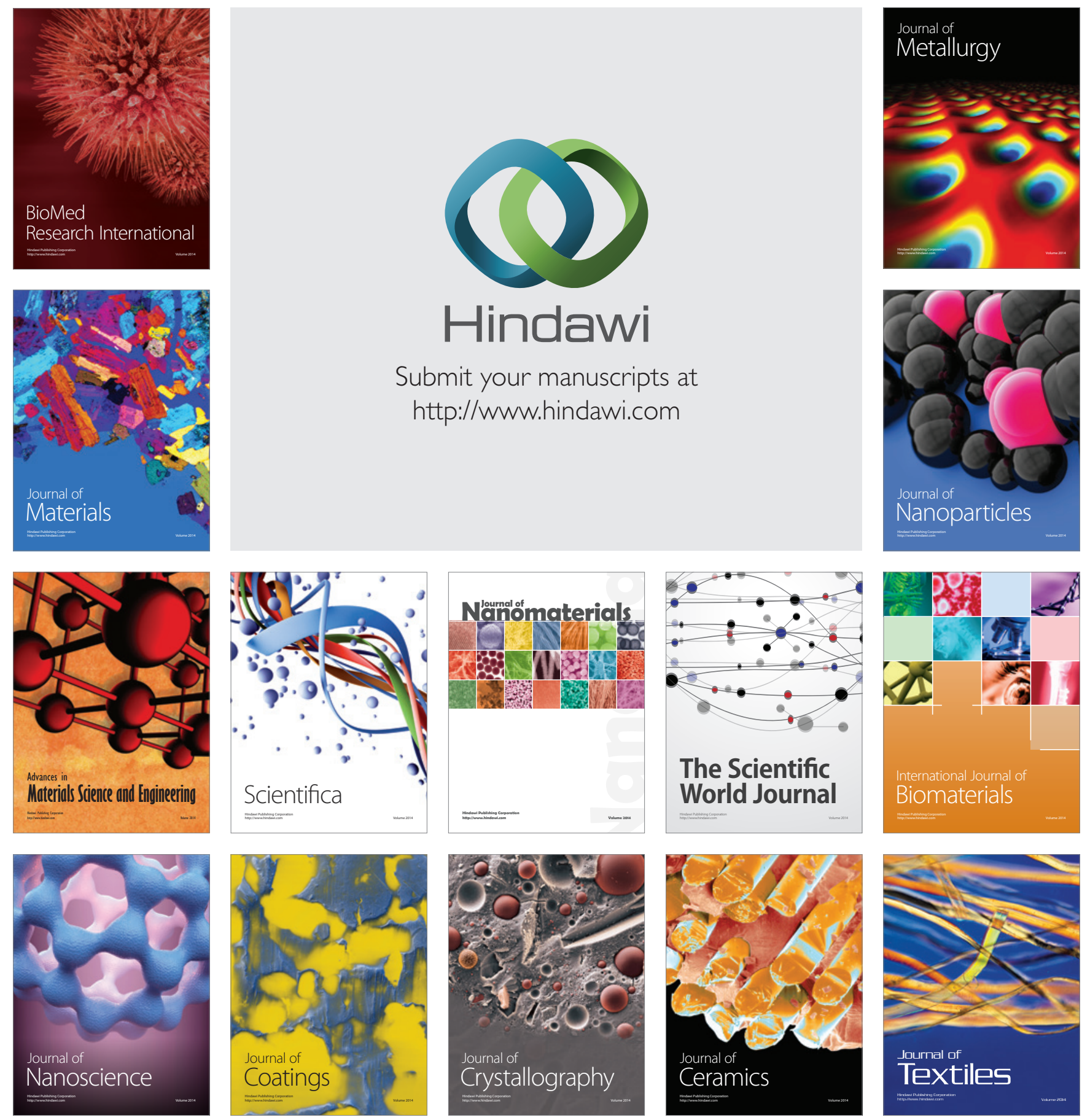